\title{
Looking for Outcomes: The Experience of Control and Sense of Agency in Obsessive- Compulsive Behaviors
}

S. Belayachi

\section{1 \\ Introduction}

Obsessive-compulsive disorder (OCD) is, as its name implies, characterized by obsessions (i.e., recurrent thoughts or images, particularly ones that cause distress) as well as compulsions (i.e., urges to perform mental or physical acts repeatedly), both of which significantly impair everyday functioning [1]. Obsessions are considered to be recurrent distressing impressions that something is wrong with an action or with a situation, such as an error or an imminent danger [2-4]. Compulsions are generally conceptualized as aiming to prevent feared harmful events and are thus associated with an increased sense of responsibility [5, 6]. However, they may also be motivated by particular sensory experiences concerning actions, such as feelings of incompleteness, that trigger the need to adjust them, rather than the avoidance of potential harm [7].

Both the sense of responsibility and the feeling of incompleteness can be viewed as experiences of actions that are obviously in conflict with the actual action context. Specifically, compulsions can be seen as behavioral responses to recurrent feelings of dissatisfaction regarding an intended achievement. Moreover, OCD features have been consistently connected to deficits affecting action processing, such as action planning [8,9] and action monitoring [10, 11].

Research on the sense of agency has highlighted the importance of action specification (e.g., outcome anticipation) and action monitoring (e.g., assessing the degree of concordance between anticipated and actual outcomes) in the subjective understanding of "what one is doing" and "what one is causing" [12-17]. Hence, a dysfunction affecting these components of action processing could lead to an incon-

S. Belayachi $(\bowtie)$ 
sistent appraisal of one's actions and of the surrounding environment. More importantly, recent models of sense of agency suggest that there are various levels of action specification and monitoring (e.g., sensorimotor, perceptual, and conceptual levels); each level may contribute to a specific component of the sense of agency [14, 18]. Therefore, the nature of the impairment and the level at which the dysfunction applies may underlie specific patterns of a defective sense of agency.

In this chapter, we review the phenomenology of OCD to understand how its heterogeneity can be depicted as an outcome processing issue and how it can be differentially affected across distinct OCD profiles.

\section{2}

\section{The Clinical Features and Phenomenology of OCD}

Obsessive-compulsive disorder is commonly viewed as a set of maladaptive habits and ways of thinking, of which obsessions and compulsions are the most prominent symptoms. As in other psychopathological states, OCD symptoms are present to some degree in most people; their frequency and their impact on everyday functioning may distinguish non-clinical from clinical cases of OCD [19]. Obsessions involve intrusive thoughts, images, or impulses that cause significant distress. Common obsessions include preoccupation with contamination, concerns about potential threatening outcomes, fear of harming oneself or others, repeated doubts about selfaction, and preoccupation with action satisfaction. Over $90 \%$ of patients with OCD report performing compulsive behaviors to reduce the distress associated with obsessions [20]. Compulsions refer to the need to perform mental or physical acts in a repeated or stereotyped way. Repetitive checking (e.g., checking locks, lights, and appliances) and repetitive washing (e.g., hand washing, house cleaning) are the most common compulsions $[21,22]$.

An important feature of OCD is that some behaviors and activities that are performed automatically by most people (e.g., washing one's hands, locking a door, tidying up clothes) are related to a dysfunctional experience of action. Examples include an inflated sense of responsibility for the occurrence or avoidance of bad outcomes, beliefs that one's thoughts can have direct negative consequences for the external world, beliefs that errors can have harmful consequences, an exaggeration of the probability and severity of potential harm, inconsistent impressions of failure or feelings of imperfection, feelings that actions or intentions have been incompletely executed, and an undermined sense of goal satisfaction. These inconsistent feelings and beliefs form strong motivational features concerning the occurrence of OCD symptoms. They can be classified according to the extent to which OCD symptoms are predominantly characterized by feelings of responsibility or of incompleteness.

Responsibility in OCD refers to the "belief that one has power which is pivotal to bring about or prevent subjectively crucial negative outcomes" ([23], p. 111). This OCD dimension seems to be dominated by cognitive phenomena, including dysfunctional beliefs, biased inferences and judgments, misinterpretation of intrusive 
thoughts. One important feature of this phenomenon is the premonitory aspect of negative outcomes. Indeed, OCD individuals experience the content of certain intrusive thoughts as an indication of a future negative consequence of their action or inaction. They may also voluntarily try to foresee a wide range of negative consequences of their actions. In addition, the processing of anticipated negative outcomes is influenced by dysfunctional beliefs, such as the idea that doing nothing about potentially upcoming bad events is equivalent to causing disastrous outcomes. Overall, these features lead OCD individuals to experience feelings of responsibility and guilt and compel them to undertake preventive actions (i.e., avoidance strategies).

On the other hand, incompleteness and "not just right" feelings are dominated by sensory phenomena and are described by patients as impressions of failure or feelings of imperfection. Such feelings can lead to an inability to achieve a sense of "task completion" or "closure" regarding actions (e.g., locking the door) or perceptions (e.g., objects on a table). This sense of dissatisfaction may cause people to experience inconsistent feelings that "actions or intentions have been incompletely achieved" ([24], p. 80). It may also lead them to feel only a weak sense of goal satisfaction. Repeated compulsions may then be motivated by the need to alleviate feelings of incompleteness or to feel "just right" [25].

Although these two distinct motivational core features may be more fundamental than mere symptom clusters, their prevalence seems to vary across the different OCD subtypes. Indeed, harm avoidance may particularly be reflected in washing and obsessing symptoms [26]. Incompleteness, on the other hand, may be especially associated with checking [26, 27] and ordering [26, 28]. Furthermore, distinct neural correlates have been associated with these different OCD subtypes. For example, checking symptoms, which are frequently related to incompleteness, may be connected to increased activity in brain regions involved with motor processing, such as dorsolateral prefrontal regions, putamen/globus pallidus, and brainstem nuclei [29-31]. Washing symptoms, which are related to harm avoidance, have been found to be associated with increased activity in brain regions that process emotional aspects of information (e.g., orbitofrontal regions [29, 31]).

Overactive performance monitoring, as reflected by the anterior cingulate cortex hyperactivity and electrophysiological abnormalities observed in OCD, has consistently been connected to incompleteness features [11,32-34]. It is assumed to reflect excessive error detection, caused by an impaired comparison between actual and expected responses $[35,36]$. Indeed, an internal comparator mechanism may compare the internal representation of action and the resulting action. If a conflict is detected, the system triggers a signal and adjustment behavior is activated. It has been suggested that a hyperactive error signal in OCD arises from a dysfunction in a comparator mechanism, which then erroneously detects a mismatch between representations of the actual and the intended response.

On the other hand, responsibility features seem to be associated with increased activity in brain regions that process the emotional aspects of action, such as the orbitofrontal cortex (OFC). The OFC may play an important role in action control and guidance of behaviors, through outcome representations and particularly by anticipating the affective impact of outcomes. This form of anticipation plays an 
9 important role in decision-making [37]. The representation of anticipated outcomes may depend on how the OFC generates possible alternative outcomes of one's action. Moreover, lateral areas of the OFC may underlie the anticipation of potential negative outcomes, while areas from the ventral and medial prefrontal cortex may be specifically involved in representing the impact of outcomes with a positive valence $[38,39]$. Increased activity in the lateral OFC in OCD individuals has been consistently related to their concerns with potential future negative outcomes [40].

Taken together, the above-mentioned studies suggest that OCD symptoms are related either to an impaired ability to re-integrate generated outcomes as being consistent with intended outcomes, or to an increased processing of outcomes, particularly of their emotional value at an early stage of action specification. From this perspective, compulsions can be conceptualized as behavioral strategies aimed at either generating outcomes that match the intended ones, as is the case in incompleteness phenomena, or avoiding the occurrence of potential negative consequences.

To sum up, people with OCD may experience a sense of responsibility for threatening events whose occurrence are not related to their actions. Yet, these individuals feel compelled to deploy compulsions in order to counter bad outcomes. Other OCD individuals, however, may perform compulsive behaviors because they have inconsistent feelings that an action has not been satisfactorily completed or that their goals and intentions have not been achieved. Compulsive behaviors related to incompleteness phenomena have the purpose of generating outcomes that will provide a sense of task completion. Furthermore, the phenomenal state preceding compulsions can be related either to "feelings of undesired end-state being achieved" or to "feelings of desired end-state being unachieved." The former may trigger avoidance strategies while the latter may trigger adjustment behaviors.

In both cases, dysfunctional outcome processing seems to be implicated in the inconsistent experience of action. For example, neurobiological studies indirectly suggest that incompleteness phenomena are related to an impaired ability to perceive generated outcomes as being consistent with their internal representations; whereas harm avoidance and responsibility may be related to an increased focus on potential negative outcomes of action. Nevertheless, outcome processing plays an important role in the experience of action, especially the mechanism that compares events appearing upstream from action (i.e., outcome anticipation) with those occurring downstream (i.e., generated outcomes) [12-17]. Hence, theories concerning sense of agency offer a reliable context to understand the dysfunctional construal of selfaction in OCD.

9.3

\section{Sense of Agency in OCD: Empirical Data}

Several models of OCD highlight a possible disturbance affecting the experience of control over one's action or events. For example, Shapiro [41] suggested that compulsions in OCD reflect a diminished inner feeling of control (sense of autonomy), lead- 
ing patients to monitor their actions with a conscious effort. OCD individuals consistently self-report experiencing a diminished sense of control in everyday life (i.e., self-perception of one's ability to attain or avoid specific outcomes through one's actions) in several studies [42-44]. However, other studies showed that they can experience a high sense of control [45] or have a higher need for control [46]. These conflicting results have been interpreted as reflecting the fact that, in response to their undermined sense of control, some OCD individuals may deploy compulsions to regain control over their actions or over unwanted events [47, 48]. From this perspective, compulsive behaviors can be viewed as a way of artificially inflating affected individuals' feelings of control, when the mechanism underlying a "naturally occurring" sense of control breaks down $[49,50]$.

The experience of control is an important component of the sense of agency, which depends on how an individual links an action to external outcomes [14]. An action control mechanism may be crucial for assessing the extent to which one's actions produce the desired or expected outcomes; the process that compares the representation of expected or desired outcomes to observed outcomes may be the most important one in this context [12-17]. In everyday actions, people do not consciously compare "what they intended to do" with "what they actually did"; they only need to access the results of the unconscious comparison, that is, a matching signal [12, 14]. However, mismatch signals may occur when the automatic action control fails to guide and monitor an action until goal attainment (which may occur in everyday behavioral situations in most people from time to time). This, then, causes the automatic control to be passed back to a conscious monitoring of action in order to secure goal attainment $[14,49]$. However, if the conscious control also fails to guide actions until the desired end-state, then it is more appropriate to abandon conscious monitoring and even to momentarily set aside the pursuit of this goal. A recent theoretical suggestion posits that, by contrast, OCD individuals are characterized by an inability to relax inefficient conscious action monitoring, leading to the deployment of a range of ways to achieve their goals [49]. To resume, the characteristic feature of OCD is an abnormally low sense of control that may be compensated through compulsive behaviors, which may have the effect of creating (artificially) a subjective experience of control. From this perspective, OCD can be reasonably viewed as a disturbance of the experience of the control component of the experience of action. The studies presented in this section provide a deeper understanding about the potential defective sense of agency in OCD, through impaired action control mechanisms.

The way in which individuals with OCD symptoms understand the relationship between their actions and their related outcomes has been examined in the context of action identification theory $[51,52]$. This theory posits that any behavior can be identified within a cognitive hierarchy of meanings. Higher-level meanings relate to the desired goal and expected outcomes; lower-level meanings, however, represent instrumental features and movement parameters. Vallacher and Wegner [51, 52] suggested that the particular level at which an action is identified reflects the particular representation (movement parameters vs. outcome) on the basis of which the action is conducted and monitored. Dar and Katz [53] explored the level at which patients with washing symptoms identified the habitual act of washing their hands, compared 
to non-OCD controls. In their study, the authors used an item related to washing symptoms (i.e., habitually washing hands). This act was associated with 22 items varying in their level of abstraction (11 low-level items such as "I run water over my hands"; 11 high-level items such as "I show responsibility to myself"). Patients and non-OCD controls had to indicate their degree of agreement with each item. Their results suggested that patients conduct their rituals with a representation of goals and outcomes that are too abstract (e.g., "I clean myself internally," "I show responsibility to myself") compared to non-OCD controls. Furthermore, this study highlighted the unusual purpose and outcomes that are related to such a basic action. The authors suggested that OCD patients' unusual representation of the act of hand washing is related to their attempts to control potential harm. Clearly, there is no specific action plan that allows one to avoid general threatening events and disasters (e.g., preventing a fire that may destroy the building). In OCD, this fact is compensated by associating a basic action with idiopathic cues for safety; compulsions are then deployed until those cues are encountered (e.g., a specific internal state such as diminished anxiety); the identification of those cues as outcomes of one's action may then provide a feeling (albeit illusory) that one's actions can control meaningful life events.

Although the way an action is identified depends on several action-related features (e.g., action complexity, degree of expertise, action disruption or error), people tend to adopt a predominant level of action identification across behaviors (i.e., level of agency [52]). Thus, the level of agency refers to the preferential level at which actions are generally identified. People with a low level of agency tend to focus on movement parameters, including sensorimotor consequences of actions, and people with a high level of agency focus on abstract goals and on the implications of behaviors. Belayachi and Van der Linden [54] examined the relationship between OCD symptoms (i.e., checking and washing symptoms) and the level of agency (i.e., the preferential level of action identification) in non-clinical participants. In this study, participants were presented with 23 items, each of which consisted of an everyday action (e.g., locking a door) followed by two alternatives or "identities." One was a low-level depiction of the action (e.g., putting a key in the lock) and the other depicted the action at a high level (e.g., securing the house). Participants had to choose the alternative that best described each action. The results suggested that checking symptoms were related to a general tendency to identify actions mainly in terms of their procedural aspects and motor components, rather than according to the related outcomes. This is in agreement with the idea that doubts about the performance and repetition of action (which characterize checking) are related to the focus of "attention to low-level gestural units of behavior rather than to goal-related higher-level units that are normally used in action flow parsing" ([55], p. 1). Furthermore, Vallacher and Wegner [52] showed that focusing on movements during an action, and not on the goal, might impair action regulation by promoting abnormal "signals of inconsistency and error," particularly during routine actions. Overall, this fits with the idea that OCD symptoms related to incompleteness phenomena (i.e., checking) are connected with an impaired action monitoring mechanism that may inconsistently generate mismatch signals or that is not able to generate a matching signal [56]. In addition, the low level of agency was specifically related to checking symptoms, as compared to 
washing symptoms, which were not related to any particular level of agency. Thus, the high level of action identification observed in the Dar and Katz study seems to be specific to patients' related concerns and compulsions.

Two studies directly examined the sense of control component of sense of agency in OCD symptoms. First, Reuven-Magril, Dar, and Lieberman [50] investigated the potential relationship between the illusory experience of control, compulsive-like behavior, and OCD symptoms in both non-clinical participants and OCD patients. They used a preprogrammed sequence of neutral and aversive images for this purpose. In this task, the participants had to attempt to control and shorten the duration of the image presentation (i.e., desired outcome) by finding the right combination of key presses (i.e., action). Their perceived level of control was assessed at various points during the task. Participants did not have any actual control over the duration of the stimuli. Indeed, the presentation time gradually increased (i.e., increased discrepancy between desired and actual outcomes) throughout the first half of the task and then gradually decreased for the remaining stimuli (i.e., increased matching between desired and actual outcomes). The results showed that OCD symptoms in both clinical and non-clinical populations (regardless of OCD subtype) were related to more compulsive-like repetitive patterns of action (i.e., using the same key presses) and to an increased (illusory) sense of control for aversive and, to a lesser extent, neutral stimuli. The authors reasonably interpreted their results as consistent with the idea that compulsions must be viewed as conscious attempts to inflate OCD individuals' feelings of control over aversive events (i.e., an effortful control). Moreover, the relationship found between inflated sense of control for neutral stimuli and OCD symptoms has been related to the high need for control that characterizes some OCD individuals (i.e., the general motivation of being able to exert control over events). Furthermore, a higher illusory sense of control in OCD individuals suggests that they overestimate the extent to which their actions can control events. This is consistent with the phenomenology observed in cases of exaggerated threat anticipation and inflated responsibility, expressed as the "belief that one has a pivotal power to bring about or prevent subjectively crucial negative outcomes" ([23], p. 111).

In a later study, Belayachi and Van der Linden [57] specifically examined the mechanism that may be involved in the "naturally occurring" feeling of control over outcomes that match expectations, in non-clinical participants with OCD symptoms. The study was based on the assumption that an unconscious comparing mechanism that grasps a correspondence between expected and actual outcomes underlies the subjective experience of control (in effortless situations). This mechanism was explored with a task in which participants were made to feel that they cause an (actually uncontrollable) outcome, because this outcome was subliminally primed (emulating outcome anticipation) before the participants acted [12]. Subliminal priming of outcomes is thought to mimic the automatic activation of the representations of action effects before the action, while simultaneously preventing conscious awareness of these thoughts. In this task (the Wheel of Fortune task), the participant and the computer each moved a square in opposite directions. The participants' task was to press a key (i.e., move) to stop the motion of the squares (i.e., "actual outcome"), and subsequently to determine whether they or the computer caused the square to 
stop in the observed position (i.e., agency judgment). In reality, the participants had no control over the movements of the square. The outcome was arranged to not represent either the participants' or the computer's real stop position. In half of the trials, the square position to be presented was primed just before the participants stopped the motion of the square (i.e., prior thoughts about expected effect). The results showed that effect priming significantly enhanced feelings of control over the rapidly moving square, as participants experienced stronger feelings that they caused the square to stop in the presented position, on primed trials. Under these conditions, participants with checking symptoms experienced lower feelings of control in both primed and non-primed trials. This effect was found to be specific to checking, and not for the other OCD dimensions, and remained when comorbid depression was controlled for. The authors interpreted those results as being consistent with the action monitoring dysfunction hypothesis, according to which an inability to generate a consistent matching signal may lead to incompleteness phenomena. In addition, this unconscious perception of a match between expected and actual outcomes may constitute an important cue for goal satisfaction and subsequent action closure in everyday behaviors [12, 58-60]. Yet, an attenuation of this phenomenal cue may explain why checking individuals frequently experience incompleteness and doubts about the goal achievement. Thus, checking compulsions could be behavioral adjustments expressed in order to receive more convincing (explicit) cues about actual goal completion or to experience completeness (i.e., "just right" feelings). Finally, there was no association between OCD symptoms and illusory sense of control, in contrast to the link observed between illusory sense of control and OCD symptoms (regardless of OCD subtype) in the Reuven-Magril et al. study[50]. These patterns of results have been interpreted as confirming the idea that illusion of control in OCD is connected to a conscious effort to obtain such subjective effects (i.e., compulsive-like behaviors), a situation that was not allowed by the paradigm used in our study.

\section{4 \\ Summary and Discussion}

Two studies have explored the way individuals with the most representative OCD symptoms (checking and washing symptoms) construe the outcomes of their actions, by assessing the level of action identification [53, 54]. The results of both studies suggest that checking symptoms are related to a tendency to identify various common actions at a low level of action construal (i.e., according to movement parameters rather than goal and outcome aspects [54]). On the other hand, washing individuals were found to identify their highly familiar act of washing hands at a higher level than non-OCD controls (i.e., a higher level of action identification [53]), although such an outcome-related identification actually reflected the fact that, compared to non-OCD controls, patients endorsed more magical and unusual outcomes depicting washers' concerns (e.g., "I clean myself internally"). This could explain why washing symptoms were not found to be related to any particularly high level of 
identification for various habitual actions [54]. Although comparably defective action processing has been observed in both sub-clinical and clinical OCD [11, 33, $61,62]$, one could argue that the use of non-clinical participants in the Belayachi and Van der Linden study and of clinical participants in the Dar and Katz study also account for the divergent results concerning washing symptoms.

Although these data are preliminary and must be interpreted with caution, the patterns of results are rather consistent with the idea that defective outcome processing may be related to OCD, and that it may be differentially affected across OCD subtypes. Indeed, washing symptoms may be specifically motivated by harm avoidance [26], while checking symptoms may be particularly related to incompleteness experiences [26, 27]. By extrapolation, the results of the Belayachi and Van der Linden and Dar and Katz studies could suggest that incompleteness implies a lack of processing of actual outcomes generated by actions; harm avoidance, on the other hand, may be related to inconsistent processing of unrelated events that are misinterpreted as resulting from one's actions. Interestingly, the level at which action is identified may determine the extent to which people experience a feeling of control for outcomes that match expectations [63].

Consistently, the results of two studies $[50,57]$ that investigated the sense of control in persons with OCD symptoms can be interpreted along the same lines. Participants with OCD symptoms (regardless of OCD subtype) may be characterized by an increased illusory sense of control in compulsive-like situations (i.e., when their actions are directed towards effortful attempts to control an event [50]). However, only individuals with checking proneness appeared to experience an undermined sense of control in routine-like situations (i.e., when their actions are supposed to be automatically controlled, rather than under conscious monitoring [57]). This latter result is consistent with the van der Weiden et al. [63] study in which people with a low level of agency were also found to be less prone to experience the illusion in the Wheel of Fortune task. The authors suggested that these results reflect situations in which people have an intention to generate a specific outcome but lose track of this intention in the course of action because they did not keep a high-level representation as they monitored the action at a lower level; consequently, they may lack experience of control and agency for the intended outcomes they have yet produced. Overall, a chronic low-level of agency [54] and related undermined sense of agency [57] in individuals with checking symptoms could explain the repeated enactment of routine actions regardless of the obvious achievement of the goal.

In the study of van der Weiden et al. [63], people with a high level of action identification were found to be more prone to experience the illusion of control. This is consistent with the illusion of control in OCD individuals observed in the ReuvenMagril et al. study, but not with the absence of association between OC symptoms and an increased experience of control in the Belayachi and Van der Linden study (i.e., illusion of control, as measured by using the Wheel of Fortune task). It is rather difficult to compare these studies as the task used in each one differed in terms of the kind of experience of control it assessed. Indeed, a recent article on the contribution of sense of control to sense of agency proposed to distinguish between the "sense that one has to exert control to generate and maintain an appropriate action program" and 
9 the "sense that one feels in control of an action" ([14, p. 20). Accordingly, the basic "sense that one feels in control of an action" only requires access to the result of the unconscious comparisons between predicted and actual states ("effortless control as for routine or automatic actions" [14]. On the other hand, the importance of mental and behavioral attempts and the adjustments necessary to reduce the discrepancies between expectations and outcomes may underlie a distinct form of experience of control (i.e., "sense that one has to exert control to generate and maintain an appropriate action program" as would be the case in disrupted or unfamiliar actions). In such effortful situations, the conscious effort itself may enhance the impression that one is engaged in and causing actions.

In light of this theoretical framework, we could argue that the illusion of control task used in the Belayachi and Van der Linden study elicited a sense of effortless control (i.e., predominantly based on automatic and unconscious processes of comparison), similar to that observed during non-conscious goal pursuit [12, 13]. Interestingly, no OCD symptoms were found to be correlated with an increased experience of control; moreover, only the checking symptoms were related to an undermined sense of control. This result is in agreement with a recent study in which selfreported low sense of control was found to be particularly related to checking symptoms [48]. In the task used by Reuven-Magril et al. [50], feelings of control were predominantly elicited by the effortful control situation created by the design of the task. Overall, this is consistent with a recent theoretical suggestion that compulsions are conscious attempts to regain control over action when automatic action monitoring fails to guide actions to the desired outcome (e.g., when the goal is too abstract [49]).

Thus, some empirical evidence seems to confirm Salkovskis's [23] assumption that harm avoidance and inflated responsibility might be construed as an illusory "perception of self-agency" It should be noted that the illusory sense of control reported by Reuven-Magril et al. [50] may highlight the phenomenon whereby compulsive-like behaviors allow OCD individuals to regain control over unwanted outcomes (e.g., aversive image) rather than the phenomenon in which they need to control, and they experience responsibility for anticipated outcomes. Indeed, a key feature of harm avoidance and responsibility phenomena is the exaggerated anticipation of negative outcomes and the belief that doing nothing to avoid those outcomes is similar to causing premeditated harm.

Interestingly, perceived premeditation may stem from any anticipation-like mental content (such as foresight, effortful forethought, wishful thinking, and the consideration of multiple possible outcomes of action). Therefore, such actions would lead people to feel responsible for those outcomes and to think that they are under personal control [64]. This counterfeit perception of self-responsibility and personal control may occur despite the obvious irrelevance of premeditation and overt behaviors, and despite the absence of any causal relationship between premeditation and observed outcomes [64]. For example, non-clinical participants can experience control and agency for observed outcomes that match prior conscious thoughts, even when the causation appears to be magical and when the thought-about outcome is viewed as undesirable [65]. Thus, future studies should explore the relationship between harm and responsibility phenomena, and the overestimation of personal influence in the 
occurrence of magical and/or threatening outcomes in a laboratory context.

As for incompleteness, the possible involvement of an impaired unconscious comparison mechanism fits with the idea that "not just right" experiences and incompleteness form a fragmented subjective experience resulting from the inability of action monitoring to generate consistent matching signals [56]. An alternative explanation of Belayachi and Van der Linden's [57] results is that checking, which is related to incompleteness phenomena, is linked to an abnormal access to motor and proprioceptive signals, due to low-level action identification (i.e., a predominant focus on sensorimotor features). Those signals are normally filtered and not consciously perceived [66-68]. Indeed, those signals play a minor role in our experience of action when we naturally focus on abstract outcome information allowing for goal achievement. However, a predominant focus on movements (i.e., low level of agency) may lead the experience of action to be mainly based on proprioceptive information [14, 69].

Conversely, it is possible that OCD individuals who feel a sense of incompleteness have an undamaged comparator mechanism, but their ability to interpret matching signals at a cognitive level is impaired [e.g., 59]. This may prevent them from feeling success when the expected outcome is achieved. It has consistently been demonstrated that priming knowledge of success alone can increase feelings of control to the same extent as priming of action effects [70]. Hence, it would be interesting to see whether priming the concept of success increases feelings of completeness for checking individuals. Future studies should explicitly explore this possibility in OCD individuals who experience incompleteness phenomena.

\section{5}

\section{Conclusion}

The symptoms of OCD can be classified according to the extent to which they are dominated by an increased sense of responsibility for random negative events (i.e., harm avoidance) or by peculiar sensory phenomena, preventing one from experiencing a sense of task completion (i.e., incompleteness). Accordingly, OCD individuals may re-enact certain actions until they obtain an outcome that informs them that they have achieved control over potential harm or until they integrate the generated outcome as consistent with the intended one. Overall, the data are consistent with the assumption that the concerns of OCD individuals regarding harm avoidance and inflated responsibility are related to an illusory "perception of self-agency" [23]. Those with incompleteness features may be characterized by an undermined sense of control, in connection with a comparator dysfunction $[11,56]$.

Throughout this chapter, we have tried to demonstrate how OCD can be reasonably understood as a disturbance of agency and to point out that the distinct patterns of the phenomenology of action and underlying mechanisms must be carefully studied. In both the incompleteness and harm avoidance phenomena, it is the way in which expected outcomes are compared to observed outcomes that may underlie the impaired experience of control. However, impaired unconscious comparisons could be 
related to a low feeling of control in incompleteness; in harm avoidance, on the other hand, dysfunctional beliefs and cognitive-related dysfunctions may account for an undermined sense of control, which also entails conscious attempts to regain control.

\section{References}

1. American Psychiatric Association (1994) Diagnostic and statistical manual of mental disorders (4th edn) American Psychiatric Association, Washington DC

2. Aouizerate B, Guehl D, Cuny E, Rougier A, Bioulac B, Tignol J, Burbaud P (2004) Pathophysiology of obsessive-compulsive disorder. A necessary link between phenomenology, neuropsychology, imagery and physiology. Prog Neurobiol 72:195 - 221

3. Schwartz JM (1998) Neuroanatomical aspects of cognitive-behavioural therapy response in obsessive-compulsive disorder. An evolving perspective on brain and behaviour. $\mathrm{Br} \mathrm{J}$ Psychiatry Suppl 38-44

4. Schwartz JM (1999) A role of volition and attention in the generation of new brain circuitry. Toward a neurobiology of mental force. J Consciousness Stud 6:115-142

5. Rachman S (1997) A cognitive theory of obsessions. Behav Res Ther 35: 793-802

6. Salkovskis P M (1985) Obsessional-compulsive problems: A cognitive-behavioural analysis. Behav Res Ther 23: 571-583

7. Summerfeldt LJ (2004) Understanding and treating incompleteness in obsessive-compulsive disorder. J Clin Psychol 60:1155-1168

8. Cavedini P, Riboldi G, D'Annucci A, Belotti P, Cisima M, Bellodi L (2002) Decision-making heterogeneity in obsessive-compulsive disorder: ventromedial prefrontal cortex function predicts different treatment outcomes. Neuropsychologia 40: 205-211

9. Veale DM, Sahakian BJ, Owen AM, Marks IM (1996) Specific cognitive deficits in tests sensitive to frontal lobe dysfunction in obsessive-compulsive disorder. Psychol Med 26: $1261-1269$

10. Pitman RK (1987) A cybernetic model of obsessive-compulsive pathology. Compr Psychiatry 28: 334-343

11. Gehring WJ, Himle J, Nisenson LG (2000) Action monitoring dysfunction in obsessive compulsive disorder. Psychol Sci 11: 1-6

12. Aarts H, Custers R, Wegner DM (2005) On the inference of personal authorship: Enhancing experienced agency by priming effect information. Conscious Cogn 14: 439-458

13. Aarts H, Wegner DM, Dijksterhuis A (2006) On the feeling of doing: Dysphoria and the implicit modulation of authorship ascription. Behav Res Ther 44: 1621-1627

14. Pacherie E (2008) The phenomenology of action: A conceptual framework. Cognition 107: $179-217$

15. Wegner DM (2002) The illusion of conscious will. MIT Press, Cambridge

16. Wegner DM, Sparrow B (2004) Authorship processing. In : Gazzaniga M (ed) The new cognitive neurosciences (3rd ed). MIT Press, Cambridge, pp 1201-1209

17. Wegner DM, Wheatley TP (1999) Apparent mental causation: Sources of the experience of will. Am Psychol 54: 480-492

18. Jeannerod M (2009) Le Cerveau volontaire. Éditions Odile Jacob, Paris

19. Rasmussen SA, Eisen JL (2002) The course and clinical features of obsessive compulsive disorder. In: Davis KL, Charney D, Coyle JT, Nemeroff C (eds) Psychopharmacology: a fifth generation of progress. Williams and Williams, Philadelphia

20. Foa EB, Kozak MJ (1995) DSM-IV field trial: obsessive-compulsive disorder. Am J Psychiatry 152:90-96 
21. Rasmussen S, Eisen J (1994) The epidemiology and clinical features of obsessive-compulsive disorder. Psychiatr Clin North Am 15: 743-758

22. Skoog G, Skoog I (1999) A 40 year follow-up of patients with obsessive-compulsive disorder. Arch Gen Psychiatry 56 : 121-127

23. Salkovskis PM (1996) Cognitive-behavioral approaches to the understanding of obsessional problems. In : Rapee RM (ed) Current controversies in the anxiety disorders. Guilford, New York, pp 103-133

24. Summerfeldt LJ, Huta V, Swinson RP (1998) Personality and obsessive-compulsive disorder. In: Swinson RP, Antony MM, Rachman S, Richter MA (eds) Obsessive-compulsive disorder: Theory, research, and treatment. Guilford, New York, pp 79-119

25. Prado HS, Rosário MC, Lee J, Hounie AG, Shavitt RG, Miguel EC (2008) Sensory Phenomena in Obsessive-Compulsive Disorder and Tic Disorders: A Review of the Literature. CNS Spectr 5: 425-432

26. Tolin DF, Brady RE, Hannan S (2008) Obsessional beliefs and symptoms of obsessive compulsive disorder in a clinical sample. J Psychopathol Behav Assess 30: 31-42

27. Coles ME, Frost RO, Heimberg RG, Rhéaume J (2003) "Not just right experiences": Perfectionism, obsessive-compulsive features and general psychopathology. Behav Res Ther 41: 681-700

28. Ecker W, Gönner S (2008) Incompleteness and harm avoidance in OCD symptom dimensions. Behav Res Ther 46: 895-904

29. Mataix-Cols D, Cullen S, Lange K, Zelaya F, Andrew C, Amaro E et al. (2003) Neural correlates of anxiety associated with obsessive-compulsive symptom dimensions in normal volunteers. Biol Psychiatry 53:482-93

30. Mataix-Cols D, Wooderson S, Lawrence N, Brammer MJ, Speckens A, Phillips ML (2004) Distinct neural correlates of washing, checking, and hoarding symptom dimensions in obsessive-compulsive disorder. Arch Gen Psychiatry 61: 564-76

31. Phillips ML, Marks IM, Senior C, Lythgoe D, O’Dwyer A-M, Meehan O et al (2000) A differential neural response in obsessive-compulsive disorder patients with washing compared with checking symptoms to disgust. Psychol Med 30:1037-50

32. Fitzgerald KD, Welsh RC, Gehring WJ, Abelson J, Himle J, Liberzon I, Taylor SF (2005) Error-related hyperactivity of the anterior cingulate cortex in obsessive compulsive disorder. Biol Psychiatry 57: 287-294

33. Hajcak G, Simons RF (2002) Error-related brain activity in obsessive-compulsive undergraduates. Psychiatry Res 110: 63-72

34. Ursu S, Stenger VA, Shear MK, Jones MR, Carter CS (2003) Overactive action monitoring in obsessive-compulsive disorder. Psychol Sci 14: 347-353

35. Falkenstein M, Hoormann J, Christ S, Hohnsbein J (2000) ERP components on reaction errors and their functional significance: a tutorial. Biol Psychol 51: 87-107

36. Gehring WJ, Goss B, Coles MGH, Meyer DE, Donchin E (1993) A neural system for error detection and compensation. Psychol Sci 4: 385-390

37. Kringelbach ML (2005) The human orbitofrontal cortex: linking reward to hedonic experience. Nat Rev Neurosci 6: 691-702

38. O'Doherty J, Kringelbach ML, Rolls ET, Hornak J, Andrews C (2001) Abstract reward and punishment representations in the human orbitofrontal cortex. Nat Neurosci 4: 95-102

39. Ursu S, Carter CS (2005) Outcome representations, counterfactual comparisons and the human orbitofrontal cortex: implications for neuroimaging studies of decision-making. Brain Res Cogn Brain Res 23: 51-60

40. Ursu S, Carter CS (2009) An initial investigation of the orbitofrontal cortex hyperactivity in obsessive-compulsive disorder: Exaggerated representations of anticipated aversive events? Neuropsychologia $47: 2145-2148$

41. Shapiro D (1965) Neurotic styles. Basic Books, New York 
42. Freeston MH, Ladouceur, R (1997) What do patients do with their obsessive thoughts? Behav Res Ther 35: 335-348

43. Ladouceur R, Freeston MH, Fournier S, Rhéaume J, Dugas MJ, Gagnon F et al (2000) Strategies used with intrusive thoughts: A comparison of OCD patients with anxious and community controls. J Abnorm Psychol 109: 179-187

44. McLaren S, \& Crowe SF (2003) The contribution of perceived control of stressful life events and thought suppression to the symptoms of obsessive- compulsive disorder in both nonclinical and clinical samples. J Anxiety Disord 17: 389-403

45. Rhéaume J, Ladouceur R, Freeston MH, Letarte H (1995) Inflated responsibility in OCD: Validation of an operational definition. Behav Res Ther 33 : 159-169

46. Sookman D, Pinard G, Beck AT (2001) Vulnerability schemas in obsessive- compulsive disorder. J Cogn Psychother 15: 109-130

47. Moulding R, Kyrios M (2006) Anxiety disorders and control related beliefs: The exemplar of obsessive- compulsive disorder (OCD). Clin Psychol Rev 26: 573-583

48. Moulding R, Kyrios M (2007) Desire for control, sense of control and obsessive compulsive symptoms. Cogn Ther Res 31: 759-772

49. Liberman N, Dar R (2009) Normal and pathological consequences of encountering difficulties in monitoring progress toward goals. In: Moskowitz G, Grant H (eds) The psychology of goals. Guilford, New York, pp $277-303$

50. Reuven-Magril O, Dar R, Liberman N (2008) Illusion of control and behavioral control attempts in Obsessive-Compulsive Disorder. J Abnorm Psychol 117: 334-341

51. Vallacher RR, Wegner DM (1985) A theory of action identification. Lawrence Erlbaum Associates, Hillsdale, NJ

52. Vallacher RR, Wegner DM (1989) Levels of personal agency: Individual variation in action identification. J Pers Soc Psychol 57: 660-671

53. Dar R, Katz H (2005) Action identification in obsessive-compulsive washers. Cogn Ther Res 29: 333-341

54. Belayachi S, Van der Linden M (2009) Level of agency in sub-clinical checking. Conscious Cogn 18 : 293-299

55. Boyer P, Liénard P (2006) Why ritualized behavior? Precaution systems and action parsing in developmental, pathological and cultural rituals. Behavioral and Brain Sciences 29: 1-56

56. Coles ME, Heimberg RG, Frost RO, Steketee G (2005) Not just right experiences and obsessive-compulsive features: Experimental, self-monitoring perspectives. Behav Res Ther 43: 153-167

57. Belayachi S, Van der Linden M (in press) Feeling of doing in obsessive-compulsive checking. Conscious Cogn

58. Szechtman H, Woody EZ (2004) Obsessive-compulsive disorder as a disturbance of security motivation. Psychol Rev 111: 111-127

59. Woody EZ, Lewis V, Snider L, Grant H, Kamath M, Szechtman H (2005) Induction of compulsive-like washing by blocking the feeling of knowing: An experimental test of the security-motivation hypothesis of obsessive-compulsive disorder. Behav Brain Funct 1: 1-11

60. Woody E, Szechtman H (2000) Hypnotic hallucinations and yedasentience. Contemp Hypn 17: 26-31

61. Ecker W, Engelkamp J (1995) Memory for actions in obsessive-compulsive disorder Behav Cogn Psychother 23: 349-371

62. Zermatten A, Van der Linden M, Larøi F, Ceschi G (2006) Reality monitoring and motor memory in checking-prone individuals. J Anxiety Disord 20: 580-596

63. van der Weiden A, Aarts H, Ruys K (2010) Reflecting on the action or its outcome: Behavior representation level modulates high level outcome priming effects on self-agency experiences. Conscious Cogn doi:10.1016/j.concog.2009.12.004 
64. Morewedge CK, Gray K, Wegner DM (in press) Perish the forethought: Premeditation engenders misperceptions of personal control. In: Hassin R, Ochsner K, Trope Y (eds) Selfcontrol in society, mind, and brain. Oxford University Press, New York

65. Pronin E, Wegner DM, McCarthy K, Rodriguez S (2006) Everyday magical powers:The role of apparent mental causation in the overestimation of personal influence. J Pers Soc Psychol 91: 218-231

66. Blakemore S-J, Wolpert DM, Frith CD (2002) Abnormalities in the awareness of action. Trends Cogn Sci 6: $237-242$

67. Fourneret P, Jeannerod M (1998) Limited conscious monitoring of motor performance in normal subjects. Neuropsychologia 36: 1133-1140

68. Frith CD (2005) The self in action: Lessons from delusions of control. Consciousness Cogn 4: $752-770$

69. Wohlschläger A, Engbert K, Haggard P, Clark S, Kalogeras J (2003) Intentionality as a constituting condition for the own self and selves. Conscious Cogn 4: $708-716$

70. Aarts H (2007) Unconscious authorship ascription: The effects of success and effect-specific information priming on experienced authorship. J Exp Soc Psychol 43: 119-126 
Cap. 9 24-03-2010 16:03 Pagina 172

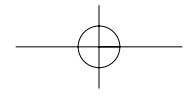

